\title{
Factors associated with the initiation of breast-feeding by Aboriginal mothers in Perth
}

\author{
C Binns ${ }^{1, *}$, D Gilchrist ${ }^{2}, M$ Gracey ${ }^{1}, M$ Zhang $^{1}$, J Scott ${ }^{3}$ and A Lee ${ }^{1}$ \\ 'School of Public Health, Curtin University of Technology, GPO Box U1987, Perth, Western Australia 6845, \\ Australia: ${ }^{2}$ Goldfields South-East Health Region, Australia: ${ }^{3}$ Division of Developmental Medicine, University of \\ Glasgow, UK
}

Submitted 18 December 2003: Accepted 4 May 2004

\begin{abstract}
Objectives: To identify the prevalence of breast-feeding at discharge and the determinants of breast-feeding initiation amongst Aboriginal women.

Design: A prospective cohort study using a self-administered baseline questionnaire and telephone-administered follow-up interviews.

Setting: Six hospitals with maternity wards in Perth, Western Australia.

Subjects: Four hundred and twenty-five Aboriginal mothers of newborn infants.

Results: At discharge, $89.4 \%$ of Aboriginal mothers were breast-feeding. Breastfeeding at discharge was most positively associated with perceived paternal support of breast-feeding, with an adjusted odds ratio (OR) of 6.65 (95\% confidence interval (CI) 2.81-15.74), and with maternal age (OR 1.12, 95\% CI 1.03-1.22), but negatively associated with parity and having delivered vaginally.

Conclusion: The factors independently associated with breast-feeding at discharge were similar to those previously identified for a group of non-Aboriginal Perth women, suggesting that separate breast-feeding interventions specially targeted at Aboriginal women are not warranted. The findings do, however, highlight the importance of including the father in the breast-feeding discussions.
\end{abstract}

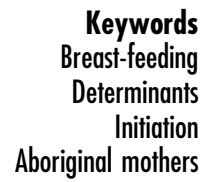

Keywords

Determinants

Initiation

Aboriginal mothers
Exclusive breast-feeding for the first six months of life is recommended for the optimum nutrition of all infants ${ }^{1,2}$. Current priorities for Aboriginal and Torres Strait Islander nutrition include an emphasis on breast-feeding ${ }^{3}$. Breastfeeding in the traditional Aboriginal communities was essential for survival of the infant and usually continued for several years ${ }^{4,5}$.

In Western Australia, differences in breast-feeding rates have been demonstrated among Aboriginal groups from various locations. Gracey et al. reported that groups in remote areas tended to continue the traditional pattern of breast-feeding for as long as possible, with 96\% of infants still being breast-fed at 12 months ${ }^{6}$. The prevalence of breast-feeding at all ages decreased with increasing urbanisation. They associated this decline with the loss of traditional culture and increasing affluence.

A study of Aboriginal mothers undertaken in Perth in the early 1980 s reported an initial breast-feeding rate of $82 \%$, declining to $50 \%$ at 3 months $^{7}$. About 15 years later in Melbourne, 98\% of Aboriginal mothers began breastfeeding but this declined to 50\% at 3 months and was down to $32 \%$ at 6 months ${ }^{8}$. Between the early 1980 s and the late 1990s there was a substantial shift in attitudes of the Australian community to breast-feeding, and the increase in the proportion of urban Aboriginal mothers initiating breast-feeding described by Holmes et al. ${ }^{8}$ might reflect the changes in the total community.

The decision to begin breast-feeding is influenced by a number of social and individual factors. A review of studies that employed multivariate analysis of the factors associated with breast-feeding initiation confirmed the importance of demographic factors such as maternal age and level of education'. There was less consistent association with factors such as marital status and socioeconomic status. Similarly, previously reported univariate associations between breast-feeding and biomedical factors such as parity, method of delivery and infant health were less consistent in multivariate studies. An intention to return to work has been shown to influence breast-feeding in some societies, particularly in Western culture $^{10,11}$. However, in other cultures, return to work was not accompanied by a decline in breast-feeding ${ }^{12,13}$.

Social support is also important in the initiation and maintenance of breast-feeding ${ }^{14}$. The Perth Infant Feeding Study (PIFS) documented the importance of the support of the father and other family members, including the maternal grandmother, in the initiation of breast-feeding ${ }^{15,16}$. 
In the 2001 National Census, 386000 persons out of a total of 18.8 million Australians were identified as Indigenous. The Indigenous population in the Perth metropolitan area at the time was reported as $18288^{17}$. The health of the Indigenous population, including morbidity and mortality related to nutritional causes, is substantially below that of other Australians ${ }^{18}$. In light of the importance of breast-feeding to the health of infants, and the lack of contemporary studies, it was decided to undertake a study of breast-feeding patterns and the determinants of breast-feeding initiation by Aboriginal women who delivered at Perth hospitals. While the few earlier studies of Aboriginal mothers have documented their breast-feeding practices, they did not attempt to identify those factors associated with the initiation of breast-feeding. If these factors differ from those identified as being important amongst non-Aboriginal women ${ }^{15,16}$, then separate breast-feeding interventions targeted specifically at Aboriginal women may be called for. The objective of this study was to identify the prevalence of breast-feeding at discharge and the determinants of breastfeeding initiation amongst Aboriginal women.

\section{Methodology}

The Perth Aboriginal Breastfeeding Study (PABS) used methodology very similar to the PIFS, conducted from September 1992 to April 1993 that has previously been published elsewhere ${ }^{15,19}$. In this current study, all Aboriginal mothers who delivered in six Perth hospitals during the survey period (April 2000 to July 2001) were contacted and invited to participate in the study. Mothers who agreed to participate in the study were interviewed in hospital and were followed up in the postpartum period. Many of the Perth hospitals provide special support for Aboriginal clients, and they make extra efforts to correctly classify the ethnicity of their patients. All mothers who identified themselves as Aboriginal were eligible to participate. However, as this study relied on selfidentification, it is possible that some Aboriginal mothers did not choose to identify themselves on admission.

The baseline questionnaire was designed to identify feeding method while in hospital and to collect information on variables known, or suspected, to be associated with breast-feeding initiation, including sociodemographic, biomedical and psychosocial factors, along with hospital practices. A number of steps were taken to ensure that the questionnaire was easy to understand. The draft of the PIFS questionnaire originally was reviewed and modified by an adult literacy expert and then pilottested on a group of 20 new mothers. Further modifications were made based on their comments. The Aboriginal nursing staff at Derbal Yerrigan Health Service also reviewed the language of the questionnaire for use in the PABS. Wherever possible, the wording of the questionnaires was kept as close as practical to the PIFS to allow for later comparison.

\section{Statistical methods}

Data were entered and analysed using the Statistical Package for Social Sciences (SPSS for Windows, version 10; SPSS Inc., Chicago, IL, USA). Unadjusted odds ratios were calculated to identify those factors that were associated with the initiation of breast-feeding. Multivariate logistic regression analysis was employed to determine which factors were independently associated with breast-feeding at discharge. Variables found to have a non-significant effect on the model were then removed in a backward stepwise fashion. All variables in the final model were variables for which, when excluded, the change in deviance compared with the corresponding chisquare test statistic on the relevant degrees of freedom was significant.

Breast-feeding terms and definitions used in this study are those recommended by The Interagency Group for Action on Breastfeeding ${ }^{20}$. The extent and duration of breast-feeding are defined by the terms full, partial and overall. Fully breast-fed infants received breast milk as their only source of milk; however, other fluids such as water or juice, or ritualistic feeds, may have been given infrequently. Partially breast-fed infants received breast milk in combination with infant formula. Overall breastfeeding is defined as receiving any breast milk.

\section{Ethics}

The purpose of the study was explained to each mother and they were asked to sign a consent form. The confidentiality of the data was assured and the mothers were assured that they could withdraw from the study at any time without prejudice. The National Health and Medical Research Council principles of research with Aboriginal communities were followed ${ }^{21}$. The project received ethical approval from the Human Ethics Committee of Curtin University, the Department of Health of Western Australia, Aboriginal Health Section and the ethics committees of the participating hospitals.

\section{Results}

A total of 455 self-identified Aboriginal mothers gave birth during the period of the study and 425 completed the baseline questionnaire while in hospital, a participation rate of $93 \%$. The sample included 441 singleton births, 13 sets of twins, and one set of triplets. The largest Perth maternity hospital contributed 310 mothers to the study, while five other hospitals provided another 115 mothers. The estimated gestational age of $15.7 \%$ of the births was 36 weeks or less and 22.6\% of infants weighed less than $2500 \mathrm{~g}$. At some time during their stay in hospital, 28.9\% of the infants spent time in a special care nursery (SCN). The Caesarean section rate for the sample was $50.4 \%$. 
At discharge from hospital $89.4 \%$ of the mothers were breast-feeding, although $4.4 \%$ were also supplementing with some formula feeds. Of the 44 mothers who were not breast-feeding, $70 \%$ had not attempted to breastfeed. The most common reasons given by mothers who did not attempt to breast-feed were a 'dislike for breastfeeding', 'bottle-feeding is easier than breast-feeding' and admission of the infant to an SCN. The rates of breast-feeding initiation were similar for infants who spent some time in an SCN and those who did not. However, of the 122 infants who were admitted to an SCN, $13.1 \%$ received some supplementary feeding compared with less than $1 \%$ of babies not requiring admission to an SCN.

Table 1 lists sociodemographic, biomedical and psychosocial factors of mother and infant that might be expected to have an influence on the establishment of breast-feeding at discharge. The numbers and percentage of breast-fed infants for each variable are given. The univariate odds ratios indicate the likelihood of a mother breast-feeding at discharge from hospital. A perceived preference of the partner and maternal grandmother for breast-feeding and whether the maternal grandmother had ever breast-fed were all positively associated with the

Table 1 Number (percentage), and univariate odds ratio (OR) and 95\% confidence interval (Cl), for breast-feeding at discharge from hospital amongst Aboriginal women in Perth $(n=425)$

\begin{tabular}{|c|c|c|c|c|}
\hline & \multicolumn{2}{|c|}{ Breast-feeding at discharge } & \multicolumn{2}{|c|}{ Univariate odds ratio } \\
\hline & Yes (\%) & No (\%) & OR & $95 \% \mathrm{Cl}$ \\
\hline \multicolumn{5}{|l|}{ Sociodemographic factors } \\
\hline \multicolumn{5}{|l|}{ Mother's age (years) } \\
\hline $14-19$ & $155(88.6)$ & $20(11.4)$ & 1.00 & \\
\hline $20-24$ & $111(88.8)$ & $14(11.2)$ & 1.02 & $0.49-2.11$ \\
\hline $25-29$ & 74 (92.5) & $6(7.5)$ & 1.59 & $0.61-4.13$ \\
\hline$\geq 30$ & $39(95.1)$ & $2(4.9)$ & 2.51 & $0.56-11.19$ \\
\hline \multicolumn{5}{|l|}{ Mother's education (years of schooling) } \\
\hline$<10$ & $152(88.4)$ & $20(11.6)$ & 1.00 & \\
\hline$\geq 10$ & $228(90.5)$ & $24(9.5)$ & 1.25 & $0.67-2.34$ \\
\hline \multicolumn{5}{|l|}{ Marital status } \\
\hline Never married/divorced/separated & 44 (84.6) & $8(15.4)$ & 1.00 & \\
\hline Married/de facto & 335 (90.5) & $35(9.5)$ & 1.74 & $0.76-3.99$ \\
\hline \multicolumn{5}{|l|}{ Mother smoking } \\
\hline No & $122(89.7)$ & $14(10.3)$ & 1.00 & \\
\hline Yes & $288(89.6)$ & $30(10.4)$ & 0.99 & $0.50-1.93$ \\
\hline \multicolumn{5}{|l|}{ Mother employed/studying before birth } \\
\hline No & $334(90.8)$ & $34(9.2)$ & 1.00 & \\
\hline Yes & $44(88.0)$ & $9(17.0)$ & 0.50 & $0.22-1.11$ \\
\hline \multicolumn{5}{|l|}{ Location* } \\
\hline Urban & $284(88.8)$ & 36 (11.3) & 1.00 & \\
\hline Rural & 96 (92.3) & $8(7.7)$ & 1.52 & $0.68-3.39$ \\
\hline \multicolumn{5}{|l|}{ Biomedical factors } \\
\hline \multicolumn{5}{|l|}{ Parity } \\
\hline Primiparous & $258(90.9)$ & $29(9.1)$ & 1.00 & \\
\hline Multiparous & $92(86.0)$ & $15(14.0)$ & 0.62 & $0.32-1.20$ \\
\hline \multicolumn{5}{|l|}{ Vaginal delivery } \\
\hline No & 198 (94.3) & $12(5.7)$ & 1.00 & \\
\hline Yes & $182(85.0)$ & $32(15.0)$ & 0.34 & $0.17-0.69$ \\
\hline \multicolumn{5}{|l|}{ Infant birth weight $(\mathrm{g})$} \\
\hline$<2500$ & $89(92.7)$ & $7(7.3)$ & 1.00 & \\
\hline$\geq 2500$ & $292(88.8)$ & 37 (11.2) & 0.62 & $0.27-1.44$ \\
\hline \multicolumn{5}{|l|}{ Infant admitted to special care nursery } \\
\hline No & $264(88.0)$ & $36(12.0)$ & 1.00 & \\
\hline Yes & $114(93.4)$ & $8(6.6)$ & 1.94 & $0.88-4.31$ \\
\hline \multicolumn{5}{|c|}{ Psychosocial factors } \\
\hline \multicolumn{5}{|c|}{ Mother attended antenatal classes for this or previous pregnancy } \\
\hline No & $358(90.6)$ & $37(9.4)$ & 1.00 & \\
\hline Yes & $23(76.7)$ & 7 (23.3) & 0.34 & $0.14-0.84$ \\
\hline \multicolumn{5}{|l|}{ Father prefers breast-feeding } \\
\hline No or ambivalent & $172(82.7)$ & $36(17.3)$ & 1.00 & \\
\hline Yes & 208 (96.3) & $8(3.7)$ & 5.44 & $2.46-12.02$ \\
\hline \multicolumn{5}{|c|}{ Maternal grandmother prefers breast-feeding $(n=425)$} \\
\hline No or ambivalent & $209(85.7)$ & $35(14.3)$ & 1.00 & \\
\hline Yes & $171(95.0)$ & $9(5.0)$ & 3.19 & $1.49-6.80$ \\
\hline \multicolumn{5}{|c|}{ Maternal grandmother breast-fed at least one infant $(n=421)$} \\
\hline No or don't know & 76 (83.5) & $15(16.5)$ & 1.00 & \\
\hline Yes & $301(91.2)$ & $29(8.8)$ & 2.05 & $1.05-4.01$ \\
\hline
\end{tabular}

*Although all women delivered in Perth metropolitan hospitals, some women gave rural locations as their place of residence. 
initiation of breast-feeding at discharge. Having a vaginal delivery and attending antenatal classes were negatively associated with breast-feeding at discharge.

To gain further understanding of the factors associated with the initiation of breast-feeding, the factors identified in Table 1 were modelled using logistic regression; those factors that remained independently associated with breast-feeding at discharge are presented in Table 2 . Breast-feeding at discharge was positively associated with maternal age and the mother's perception that the infant's father preferred breast-feeding. It was negatively associated with parity and having a vaginal delivery.

\section{Discussion}

Breast-feeding rates for Aboriginal mothers appear to be slightly higher at discharge than for non-Indigenous mothers in Perth; $89.4 \%$ compared with $83.8 \%$ in the PIFS $^{22}$. This difference may be real or may be due to the fact that the present survey was conducted approximately eight years after the PIFS and by this time the rates for nonAboriginal mothers might also be higher. Certainly the rates are at least comparable and give no cause for concern about breast-feeding initiation rates amongst urban Aboriginal women.

There was a higher rate of admission to an SCN (28.9\%) compared with the PIFS, where the rate was $15.8 \%$. The Caesarean section rate was $50.4 \%$ compared with $17 \%$ for the non-Aboriginal women in the PIFS. The high breastfeeding initiation rate was achieved despite the high rate of low-birth-weight infants, births of shorter gestational age and the high Caesarean section rate. More of the

Table 2 Factors associated with breast-feeding at discharge after adjustment for potential confounders* $(n=416)$

\begin{tabular}{|c|c|c|c|}
\hline Variable $\dagger$ & $n$ & Odds ratio & $\begin{array}{l}95 \% \text { confidence } \\
\text { interval }\end{array}$ \\
\hline \multicolumn{4}{|c|}{ Sociodemographic factors } \\
\hline Mother's age (years) & 416 & 1.12 & $1.03-1.22$ \\
\hline \multicolumn{4}{|l|}{ Biomedical factors } \\
\hline \multicolumn{4}{|l|}{ Parity } \\
\hline Primiparous & 313 & 1.0 & \\
\hline Multiparous & 103 & 0.39 & $0.16-0.94$ \\
\hline \multicolumn{4}{|l|}{ Vaginal delivery } \\
\hline No & 204 & 1.00 & \\
\hline Yes & 212 & 0.30 & $0.14-0.64$ \\
\hline \multicolumn{4}{|c|}{ Psychosocial factors } \\
\hline \multicolumn{4}{|c|}{ Father prefers breast-feeding } \\
\hline No or ambivalent & 202 & 1.0 & \\
\hline Yes & 214 & 6.65 & $2.81-15.74$ \\
\hline
\end{tabular}

-2 log likelihood (deviance) $=225.492, \mathrm{df}=4$.

* Non-significant variables were mother's marital status, level of education and location of residence, infant birth weight, whether infant had been admitted to special care nursery, whether mother smoked, had worked in the six months before her pregnancy or had attended antenatal classes for this or a previous pregnancy, whether the mother's own mother preferred breast-feeding or had breast-fed one or more of her children.

†All variables in the final model were variables for which, when excluded, the change in deviance compared with the corresponding chi-square test statistic on the relevant degrees of freedom was significant.
Aboriginal mothers managed to breast-feed while their infants were in special care: $93.4 \%$ of infants had at least some breast-feeding, compared with $77.4 \%$ of comparable infants in the PIFS. It is an indication of the dedication of the mothers and the nursing staff to the care of their infants that such high breast-feeding initiation rates were achieved.

A surprising finding was the negative association between having had a vaginal delivery and the initiation of breast-feeding. This finding is inconsistent with the literature and most researchers have either found no association between mode of delivery and breast-feeding initiation $^{23}$ or a negative association between Caesarean delivery and breast-feeding initiation ${ }^{24}$.

With this exception, the other factors found to be independently associated with breast-feeding at discharge were similar to those identified in the earlier study of nonAboriginal Perth women ${ }^{15}$. In particular, breast-feeding initiation was strongly associated in both studies with the mother's perception that the infant's father preferred breast-feeding. Aboriginal women who perceived that their partner preferred breast-feeding were more than six times as likely to be breast-feeding at discharge than women who perceived that their partner either preferred bottle-feeding or was ambivalent about how their infant was fed. Similarly, older women were more likely to be breast-feeding at discharge than were younger women, and multiparous women were less likely than primiparous women to be breast-feeding. This is different to previous studies of Australian women, which have shown that multiparous mothers are more likely to initiate breastfeeding 9 . One possible explanation is difference in parity between PIFS and PABS mothers, where the mean parities were 1.7 and 2.7 respectively, reflecting national trends ${ }^{25}$. Indigenous mothers who had already had more than five pregnancies had lower rates of breast-feeding, perhaps reflecting the impact of frequent pregnancies.

\section{Conclusion}

The strongest predictors of breast-feeding initiation among Aboriginal women were similar to those reported for non-Aboriginal women, and suggest that separate breast-feeding promotion programmes are not warranted. These factors would, however, provide an opportunity for health promotion activities to promote breast-feeding initiation rates to even higher levels. In particular, health promotion programmes should target the opinion of fathers and younger women. Overall the breast-feeding initiation rates are high despite the high prevalence of negative factors such as low birth weight and Caesarean section. This study has documented breast-feeding initiation, and further studies are needed to document breast-feeding duration and the factors determining duration. The breast-feeding initiation rate of almost 90\% is a tribute to the mothers and the health-care personnel. 


\section{Acknowledgements}

We gratefully acknowledge the willing assistance given by the mothers in our study, the hospital staff and community health workers. Without this assistance the study would not have been possible.

\section{References}

1 Binns CW. Breastfeeding. In: Binns CW, Baghurst KI, eds. Dietary Guidelines for Children. Canberra: National Health and Medical Research Council, 2002.

2 World Health Organization (WHO). World Health Assembly Resolutions, 2001. Geneva: WHO, 2001.

3 Strategic Intergovernmental Nutrition Alliance. Eat Well Australia: A Strategic Framework for Public Health Nutrition/National Aboriginal \& Torres Strait Islander Nutrition Strategy \& Action Plan. Canberra: National Public Health Partnership, 2001.

4 Hitchcock N. Infant feeding in Australia: an historical perspective. Part 3: Australian Aborigines and recent migrants. Australian Journal of Nutrition and Dietetics 1989; 46(4): 108-11.

5 Gracey M. Maternal health, breast-feeding and infant nutrition in Australian aborigines. Acta Paediatrica Japonica 1989; 31(4): 377-80

6 Gracey M, Murray H, Hitchcock N, Owles E, Murphy B. The nutrition of Australian Aboriginal infants and young children. Nutrition Research 1983; 3: 133-47.

7 Phillips F, Dibley M. A longitudinal study of feeding patterns of Aboriginal infants living in Perth, 1980-1982. Proceedings of the Nutrition Society of Australia 1983; 8: 130-2.

8 Holmes W, Thorpe L, Phillips J. Influences on infant-feeding beliefs and practices in an urban aboriginal community. Australian and New Zealand Journal of Public Health 1997; 21(5): 504-10.

9 Scott JA, Binns CW. Factors associated with the initiation and duration of breast feeding. Australian Journal of Nutrition and Dietetics 1998; 55(2): 51-61.

10 Fein S, Roe B. The effect of work status on initiation and duration of breast-feeding. American Journal of Public Health 1998; 88(7): 1042-6.

11 Roe B, Whittington LA, Fein SB, Teisl MF. Is there competition between breast-feeding and maternal employment? Demography 1999; 236(2): 157-71.

12 Lakati A, Binns C, Stevenson M. Breast-feeding and the working mother in Nairobi. Public Health Nutrition 2002; 5(6): 715-8.

13 Grummer-Strawn L. The effect of changes in the population characteristics on breastfeeding trends in fifteen developing countries. International Journal of Epidemiology 1996; 25(1): 94-102.

14 Matich J, Sims L. A comparison of social support variables between women who intend to breast or bottle feed. Social Science \& Medicine 1992; 34: 919-27.

15 Scott JA, Binns CW, Aroni RA. The influence of reported paternal attitudes on the decision to breastfeed. Journal of Paediatrics and Child Health 1997; 33: 305-7.

16 Scott J, Landers M, Hughes R, Binns C. Factors associated with the initiation and duration of breast feeding amongst two populations of Australian women. Journal of Paediatrics and Child Health 2001; 37: 254-61.

17 Australian Bureau of Statistics (ABS). Population Census of Australia, 2001. Canberra: ABS, 2002.

18 National Health and Medical Research Council. Nutrition in Aboriginal and Torres Strait Islander Peoples. An information paper. Canberra: Commonwealth of Australia, 2000.

19 Binns CW, Scott J. Breastfeeding: reasons for starting, reasons for stopping and problems along the way. Breastfeeding Review 2002; 10(2): 13-9.

20 Labbok M, Krasovec K. Toward consistency in breastfeeding definitions. Studies in Family Planning 1990; 4: 226-30.

21 National Health and Medical Research Council (NHMRC). Guidelines on Ethical Matters in Aboriginal and Torres Strait Islander Health Research. Approved by the 111th Session of the NHMRC, Brisbane, June 1991. Canberra: Commonwealth of Australia, 1991.

22 Scott J, Binns C, Aroni R. Breastfeeding Perth - recent trends. Australian and New Zealand Journal of Public Health 1996; 20(2): 210-1.

23 Kearney MH, Cronenwett LR, Reinhardt R. Cesarean delivery and breastfeeding outcomes. Birth 1990; 17: 97-103.

24 Ever-Hadani P, Seidman DS, Manor O, Harlap S. Breast feeding in Israel: maternal factors associated with choice and duration. Journal of Epidemiology and Community Health 1994; 48: 281-5.

25 Australian Bureau of Statistics (ABS). Year Book Australia, 85th ed. Canberra: ABS, 2003. 